\title{
RELACIONES INTRAFAMILIARES Y CALIDAD DE VIDA RELACIONADA CON LA SALUD EN ADOLESCENTES MEXICANOS QUE SE AUTOLESIONAN SIN INTENCIÓN SUICIDA
}

INTRA-FAMILY RELATIONSHIPS AND HEALTH-RELATED QUALITY OF LIFE IN MEXICAN ADOLESCENTS THAT SELF-INJURE WITHOUT A SUICIDAL INTENTION

Recibido: 20 de octubre de 2017 | Aceptado: 04 de abril de 2018

DOI: 10.22199/S07187475.2018.0002.00001

YOLANDA V. ChÁVEZ-FLORES 1 ; CARLOS A. HIDALGO-RASMUSSEN 2 ; SERGIO MUÑOZ NAVARRO 3

1., 2. unIVERSIDAD DE GUAdALAJARA, Jalisco, México; 3. unIVERSIDAd DE LA Frontera, Temuco, Chile

\begin{abstract}
RESUMEN
OBJETIVO: determinar la existencia de asociación entre las relaciones intrafamiliares (RI) y la calidad de vida relacionada con la salud (CVRS) en adolescentes mexicanos que se autolesionan. MÉTODO: Diseño observacional y transversal. Participaron 545 adolescentes de ambos sexos, $58.17 \%$ mujeres y $14.49 \pm 1.7$ de edad. Se utilizó la versión mexicana del ISSIQ-A, la E.R.I. y el KIDSCREEN-52. Se aplicaron modelos de regresión logística para determinar la asociación de las RI y la CVRS, ajustando por variables control. RESULTADOS: Alta unión y apoyo aumentó la posibilidad de CVRS superior con odds ratio (OR, IC95\%) de 1.90 en bienestar psicológico y 2.33 en padres y vida familiar. Alta expresión se asoció a mayor posibilidad de CVRS superior en siete dominios con OR de 1.64 en recursos económicos hasta 5.89 en padres y vida familiar. Baja dificultad incrementó la posibilidad de CVRS superior en nueve dominios, con OR de 1.51 para bienestar psicológico hasta 3.08 en relación con los padres y vida familiar. CONCLUSIONES: Aun ajustando por variables de control, las RI se asocian a la CVRS en adolescentes mexicanos que se autolesionan. Se sugiere incluir las relaciones familiares en la evaluación y tratamiento.

PALABRAS CLAVE: Calidad de vida, relaciones familiares, conducta autodestructiva, adolescente.
\end{abstract}

\section{ABSTRACT}

OBJECTIVE: To determine the association between Intra-Family Relationship (IR) and Quality of Life (HRQoL) related to Mexican adolescents' health who self-injure. METHOD: This research follows an observational and cross-sectional design. 545 adolescents (male/female) participated ( $58.17 \%$ of women and an average age of 14.49 years old \pm 1.7 .) The Mexican version of ISSIQ-A, E.R.I. and KIDSCREEN-52 were used. Models of logistic regression were applied to determine the association between the IR and the HRQOL, adjusting for control variables. RESULTS: High union and support increased the possibilities for higher HRQoL with odds ratio (OR, IC95\%) of 1.90 in psychological wellbeing and 2.33 in parents and family life. High expression was associated with more possibilities of higher HRQD in seven domains with OR of 1.64 in financial resources until 5.89 in parents and family life. Low difficulty increased the possibility of HRQL superior in nine domains, with OR of 1.51 for psychological wellbeing until 3.08 in relation to parents and family life. CONCLUSIONS: Even adjusting control variables, the IR are associated to HRQoL in Mexican adolescents who self-injure. To include the intra-family relationships in evaluation and treatment is advised.

KEY WORDS: Quality of life, family relations, self-injurious behavior, adolescent. 
A nivel internacional, el suicidio es la tercera causa de muerte en el grupo de 15 a 19 años (Organización Mundial de la Salud, 2017). En el año 2015, 67000 adolescentes murieron a causa de suicidio o accidentalmente debido a conductas autolesivas (OMS, 2017), por lo que una conducta de riesgo relacionada con el suicidio es la autolesión no suicida (Whitlock et al., 2012), definida como el daño que una persona hace a su propio cuerpo de manera directa e intencional sin el deseo explícito de causarse la muerte (Nock, 2010). Las conductas de autolesión más frecuentes son cortarse, rascarse, golpearse, tallarse y rasparse (Zetterqvist, 2015). Diversos estudios han encontrado diferencias por sexo, con una mayor cantidad de mujeres que utilizan el corte y el daño a la piel, mientras que los hombres reportan con mayor frecuencia golpearse y quemarse (Brunner et al., 2014). Respecto a la magnitud de la autolesión, una revisión sistemática determinó que la prevalencia internacional de autolesión no suicida en población escolar de adolescentes durante el periodo 2005-2011 fue del 18\% (Muehlenkamp, Claes, Havertape, \& Plener, 2012). En México se han realizado escasos estudios sobre autolesión no suicida (Benjet et al., 2017) aunque sus resultados muestran que es un fenómeno frecuente en la población adolescente. Mayer, Morales, Figueroa y Ulloa (2016) realizaron un estudio con una muestra de 804 estudiantes entre los 12 y 17 años de edad, encontrando que el $27.9 \%$ reportaron conductas de autolesión. Albores-Gallo et al. (2014) analizaron una muestra de 533 estudiantes entre los 11 y 17 años y encontraron una prevalencia del $5.6 \%$ cuando consideraron los criterios diagnósticos del DSM-5 y una prevalencia del $17.1 \%$ cuando utilizaron la pregunta ¿te autolesionas sin la intención de quitarte la vida?.

Con relación a los factores de riesgo, estudios previos han evidenciado que la presencia y severidad de la autolesión no suicida está asociada a la calidad de la relación con los padres (Di Pierro, Sarno,
Perego, Gallucci, \& Madeddu, 2012) así como al control psicológico y conductual de los padres hacia el adolescente (Baetens et al., 2014). Más aún, el contexto familiar ha sido reconocido tanto como factor protector como de riesgo ante diferentes conductas problema en los adolescentes (Valenzuela, Ibarra, Zubarew \& Correa, 2013), tales como el consumo de alcohol, tabaco y drogas ilícitas (Jordán, Molina \& Pillon, 2009). En el presente estudio, el contexto familiar se analiza a partir de las relaciones intrafamiliares definidas como las interconexiones que se dan entre los integrantes de cada familia; se componen de tres dimensiones. Unión y apoyo, que se refiere a la tendencia a convivir y apoyarse mutuamente; expresión, que evalúa la posibilidad de comunicar verbalmente las emociones, acontecimientos e ideas en un ambiente de respeto y por último la dimensión dificultades, que se refiere a los aspectos indeseables, negativos 0 problemáticos de las relaciones familiares (Rivera \& Andrade, 2010).

Por otra parte, la CVRS es definida como un constructo multidimensional que incluye aspectos físicos, emocionales, mentales, sociales, y del comportamiento y función como son percibidos por los pacientes $u$ otros individuos. Se compone de la evaluación subjetiva de diez dominios: bienestar físico, bienestar psicológico, estado de ánimo, autopercepción, autonomía, relación con los padres y vida familiar, amigos y apoyo social, entorno escolar, aceptación social y recursos económicos (Ravens-Sieberer et al., 2014). Durante la adolescencia se han encontrado diferencias en la CVRS de acuerdo al sexo y a la edad, con puntuaciones más bajas en las mujeres y en los adolescentes de mayor edad (Meade \& Dowswell, 2016). En el contexto iberoamericano se han encontrado resultados en la misma dirección; un estudio en el que participaron 1357 adolescentes de Argentina, Brasil y Chile, mostró una disminución en las puntuaciones de CVRS con el avance de la edad, siendo más 
acentuada esta tendencia para las mujeres (Guedes, Astudillo, Morales, del Campo Vecino, \& Pires Júnior, 2014).

Además, en esta etapa pueden presentarse múltiples riesgos y contrarrestar el crecimiento físico, psicológico y social (Organización Panamericana de la Salud, 2008). En este sentido, estudios previos han analizado la relevancia del contexto familiar en la CVRS de los adolescentes con diversos padecimientos (Grey, Boland, Yu, SullivanBolyai, \& Tamborlane, 1998, Herzer, Denson, Baldassano, \& Hommel, 2011; Baña, 2015) los cuales coinciden en que el funcionamiento familiar es un importante predictor de la CVRS de los adolescentes; sin embargo, hasta nuestro conocimiento no se ha estudiado la CVRS en los adolescentes que se autolesionan sin intención suicida, afección que presenta un componente psicológico y conductual distinto de los padecimientos previamente analizados, ni el papel que desempeña el contexto familiar en el mantenimiento, disminución 0 recuperación de la CVRS en esta población de adolescentes, por lo que el propósito de este estudio fue determinar la existencia de asociación entre las relaciones intrafamiliares y la CVRS en adolescentes mexicanos que se autolesionan, ajustando por variables biosociodemográficas y uso de sustancias.

\section{MÉTODO}

\section{Diseño}

El presente es un estudio observacional y transversal.

\section{Participantes}

Participaron 545 adolescentes entre los 12 y 19 años de edad, estudiantes de escuelas públicas y privadas de nivel secundaria y preparatoria en el municipio de Ciudad Guzmán, Jalisco en México. Las seis escuelas y los grupos participantes fueron seleccionados aleatoriamente. Se llevó a cabo un muestreo aleatorio estratificado según nivel y grado escolar. Se definió el tamaño de muestra de cada estrato con afijación proporcional. De un total de 1613 estudiantes evaluados, 574 adolescentes reportaron la presencia de al menos una conducta de autolesión no suicida quienes son los sujetos de este estudio. Se eliminaron 29 casos debido a datos perdidos en las variables analizadas, por lo que se incluyó un total de 545 casos en el análisis de datos.

\section{Instrumentos}

Datos sociodemográficos. Se obtuvo información referente a la edad, el sexo, el nivel educativo, la presencia de discapacidad y el nivel socioeconómico (Rivas-Torres \& Bianchi-Águila, 1991).

Autolesión no suicida. Se aplicó la versión mexicana del Cuestionario de Impulsividad, autolesión no suicida e ideación suicida en adolescentes (Carvalho et al., 2015) (ISSIQA por sus siglas en inglés) adaptada a población mexicana por Chávez-Flores, Hidalgo-Rasmussen y González-Betanzos (2017; enviado a publicación). Es un instrumento de autoreporte con 56 ítems que evalúan impulsividad (8 ítems, $\alpha=.73$ ), autolesión no suicida ( 8 ítems, $\alpha=.71$ ), conductas de riesgo ( 6 ítems, $\alpha=.67) \mathrm{e}$ ideación suicida ( 3 ítems, $\alpha=.81$ ). Cuenta con dos subescalas para evaluar las funciones que cumple la autolesión no suicida: reforzamiento automático (24 ítems, $\alpha=.94)$ y reforzamiento social (7 ítems, $\alpha=$ .86). La confiabilidad global del instrumento en la versión mexicana fue de $\alpha=.93$. Las opciones de respuesta consisten en una escala Likert de cuatro puntos que van del 0 (nunca) al 3 (siempre). A mayor puntaje, mayor presencia de autolesión no suicida, impulsividad, conductas de riesgo o ideación suicida. Las variables de control en este estudio fueron 2 ítems de la dimensión conductas de riesgo: yo abuso del alcohol y yo abuso de medicamentos (para drogarme).

Relaciones intrafamiliares. Se utilizó la versión de 12 ítems de la escala de evaluación de las relaciones intrafamiliares 
(E.R.I) diseñada para población mexicana por Rivera y Andrade (2010). Es una escala de autoreporte para evaluar las dimensiones unión y apoyo (4 reactivos, $\alpha=.81$ ), expresión (4 reactivos, $\alpha=.88$ ) y dificultades (4 reactivos, $\alpha=.78$ ). Las opciones de respuesta están en una escala Likert con opciones de respuesta que van del 1 (totalmente en desacuerdo) a 5 (totalmente de acuerdo). A mayor puntaje, mejor percepción de las relaciones intrafamiliares. Cuenta con evidencia de validez de constructo mediante análisis factorial confirmatorio, validez clínica y validez concurrente.

CVRS. Se aplicó la versión mexicana del cuestionario KIDSCREEN-52 (Screening for and promotion of Health-Related Quality of Life in Children and Adolescents) (HidalgoRasmussen, Rajmil \& Montaño, 2014). Se puede aplicar a niños y adolescentes. Evalúa diez dominios de la CVRS: bienestar físico ( 5 ítems, $\alpha=.50$ ), bienestar psicológico ( 6 ítems, $\alpha=.83$ ), estado de ánimo (7 ítems, $\alpha=.81$ ), autopercepción ( 5 ítems, $\alpha=.66$ ), autonomía (5 ítems, $\alpha=.86$ ), relación con los padres y vida familiar ( 6 ítems, $\alpha=.86$ ), recursos económicos ( 3 ítems, $\alpha=.67$ ), amigos y apoyo social ( 6 ítems, $\alpha=.85$ ), entorno escolar ( 6 ítems, $\alpha=.84$ ) y aceptación social (3 ítems, $\alpha=.83$ ). Se contesta a partir de una escala Likert de cinco opciones que va desde nunca a siempre o de nada a muchísimo. A mayor puntaje obtenido, mejor autopercepción de calidad de vida. El cuestionario cuenta con evidencia de validez de contenido y validez de constructo.

\section{Procedimiento}

Una vez otorgado el permiso de cada institución educativa, se invitó a participar a los estudiantes, se les entregó por escrito una carta de asentimiento y se hizo llegar a los padres 0 tutor una carta de consentimiento informado. Al día siguiente, se proporcionaron los instrumentos de evaluación (ISSIQ-A, E.R.I. y KIDSCREEN52) a los adolescentes que aceptaron participar y que entregaron firmado tanto el asentimiento como el consentimiento informado. Uno de los investigadores a cargo del estudio leyó las instrucciones para contestar cada instrumento y aclaró las dudas de los participantes, quienes dentro de sus aulas y durante el horario escolar contestaron los instrumentos en un tiempo aproximado a los 40 minutos. Se garantizó el anonimato y la confidencialidad a cada participante. Se brindó un folleto informativo sobre atención psicológica gratuita a todos los participantes y fueron canalizados tres participantes que solicitaron atención psicológica. El protocolo del estudio fue aprobado por el Comité de Bioética del Centro Universitario del Sur de la Universidad de Guadalajara con el registro $\mathrm{CB} / 006 / 2017$. Asimismo, se obtuvo por escrito la autorización de la Secretaria de Educación Jalisco y de los directivos de cada una de las instituciones educativas participantes.

\section{Análisis de datos}

Se realizó un análisis descriptivo de las características sociodemográficas. Para evaluar la percepción de las relaciones intrafamiliares se obtuvo la puntuación cruda para cada participante y a partir de la mediana se clasificó a los participantes en el grupo de mejor o peor percepción de las relaciones intrafamiliares; cada dimensión se recodificó con el valor 0 la exposición a relaciones intrafamiliares de riesgo (baja unión y apoyo, baja expresión y alta dificultad) y 1 a relaciones intrafamiliares consideradas sin riesgo (alta unión y apoyo, alta expresión, baja dificultad).

La CVRS se analizó considerando un punto de corte de 42 o menos puntos para cada dominio (Revicki et al. 2006) y se recodificó con el valor 0 la calidad de vida inferior y 1 la calidad de vida superior. Las variables de control se recodificaron con el valor 0 cuando se consideró factor de riesgo para calidad de vida inferior y 1 cuando no existió la exposición a riesgo: sexo $0=$ mujer, $1=$ hombre; edad $0=12$ a 14 años, $1=15$ a 19 años; nivel socioeconómico $0=$ medio bajo $y$ 
bajo, $1=$ medio alto y alto; discapacidad $0=$ sí, 1=no; abuso de alcohol y abuso de medicamentos (para drogarse) se recodificaron con 0 las opciones de respuesta que se refieren a la presencia de esta conducta (algunas veces me ocurre, me ocurre mucho y me ocurre todo el tiempo) y 1 cuando contestaron nunca me ocurre.

Considerando estudios antecedentes sobre la variable edad en la CVRS (JiménezIglesias, Moreno, Ramos \& Rivera, 2015) y en las relaciones intrafamiliares, se aplicó la prueba Chi cuadrada $X^{2}$ con un grado de libertad y un nivel de significancia del $95 \%$ $(p<.05)$ para conocer su asociación con la edad.

Para determinar la asociación entre los dominios de CVRS como variable dependiente y las dimensiones de relaciones intrafamiliares como variables independientes, se utilizó un modelo de regresión logística múltiple considerando las variables de control sexo, edad, nivel socioeconómico, discapacidad, abuso de alcohol, abuso de drogas blandas (marihuana y sus derivados) y abuso de medicamentos. Se obtuvieron las Odds Ratio (OR) y los intervalos de confianza (IC95\%). Se utilizó el programa estadístico StataSE versión 14.

\section{RESULTADOS}

La muestra consistió en 545 adolescentes mexicanos entre los 12 y 19 años de edad, con una edad promedio de $14.49 \pm 1.71$. Los participantes de secundaria fueron en su mayoría mujeres (56.63\%), de 12 a 14 años de edad (90.61\%), de nivel socioeconómico medio bajo y bajo $(64.72 \%)$ y $\sin$ discapacidad (85.76\%). De los participantes de preparatoria el $60.17 \%$ fueron mujeres, entre los 15 a 19 años de edad (98.31\%), de nivel socioeconómico medio bajo y bajo $(69.07 \%)$ y la mayoría no reporto discapacidad (88.56\%) (Tabla 1).

TABLA 1.

Características sociodemográficas de los adolescentes mexicanos que se autolesionan sin intención suicida según nivel escolar.

\begin{tabular}{lcccc}
\hline \multirow{2}{*}{ Variables } & \multicolumn{2}{c}{ Secundaria } & \multicolumn{2}{c}{ Preparatoria } \\
\cline { 2 - 5 } & $\mathrm{n}$ & $\%$ & $\mathrm{n}$ & $\%$ \\
\hline Sexo & & & & \\
$\quad$ Hombres & 134 & 43.37 & 94 & 39.83 \\
$\quad$ Mujeres & 175 & 56.63 & 142 & 60.17 \\
Edad & & & & \\
$\quad$ 12 a 14 años & 280 & 90.61 & 4 & 1.69 \\
$\quad$ 15 a 19 años & 29 & 9.39 & 232 & 98.31 \\
Nivel socioeconómico & & & & \\
$\quad$ Medio alto y alto & 109 & 35.28 & 73 & 30.93 \\
$\quad$ Medio bajo y bajo & 200 & 64.72 & 163 & 69.07 \\
Discapacidad & & & & \\
$\quad$ Sí & 44 & 14.24 & 27 & 11.44 \\
$\quad$ No & 265 & 85.76 & 209 & 88.56 \\
\hline Nota: N=545 & & & &
\end{tabular}

Cuando se comparó la CVRS según la edad, se encontró una asociación estadísticamente significativa en el dominio bienestar físico $\left(X^{2}(1) 5.73 ; p=.017\right)$ para los hombres, en la autopercepción para las mujeres $\left(X^{2}(1) 6.45 ; p=.011\right)$ y en aceptación social tanto para hombres $\left(X^{2}(1) 9.50\right.$; $\mathrm{p}=.002)$ como para mujeres $\left(\mathrm{X}^{2}\right.$ (1) 16.2; $\mathrm{p}<.001$ ) (Tabla 2). 
TABLA 2.

CVRS superior según sexo para cada grupo de edad y sus diferencias en adolescentes mexicanos que se autolesionan sin intención suicida.

\begin{tabular}{|c|c|c|c|c|c|c|}
\hline \multirow{2}{*}{ Dominiosa $^{a}$} & \multicolumn{2}{|c|}{$12-14$} & \multicolumn{2}{|c|}{$15-19$} & \multirow{2}{*}{$X^{2}$} & \multirow{2}{*}{$p$} \\
\hline & $\mathrm{n}$ & $\%$ & $\mathrm{n}$ & $\%$ & & \\
\hline \multicolumn{7}{|l|}{ Bienestar físico } \\
\hline Hombres & 98 & 59.76 & 66 & 40.24 & 5.73 & .017 \\
\hline Mujeres & 82 & 54.30 & 69 & 45.70 & 1.98 & .159 \\
\hline \multicolumn{7}{|c|}{ Bienestar psicológico } \\
\hline Hombres & 89 & 54.27 & 75 & 45.73 & .073 & .787 \\
\hline Mujeres & 75 & 48.39 & 80 & 51.61 & .380 & .537 \\
\hline \multicolumn{7}{|c|}{ Estado de ánimo } \\
\hline Hombres & 60 & 60.00 & 40 & 40.00 & 1.92 & .165 \\
\hline Mujeres & 43 & 52.44 & 39 & 47.56 & .230 & .631 \\
\hline \multicolumn{7}{|l|}{ Autopercepción } \\
\hline Hombres & 94 & 55.95 & 74 & 44.05 & 327 & .567 \\
\hline Mujeres & 101 & 56.42 & 78 & 43.58 & 6.45 & .011 \\
\hline \multicolumn{7}{|l|}{ Autonomía } \\
\hline Hombres & 87 & 56.13 & 68 & 43.87 & .332 & .564 \\
\hline Mujeres & 81 & 48.21 & 87 & 51.79 & .540 & .462 \\
\hline \multicolumn{7}{|c|}{ Relación con los padres y vida familiar } \\
\hline Hombres & 84 & 57.14 & 63 & 42.86 & .897 & .343 \\
\hline Mujeres & 75 & 48.39 & 80 & 51.61 & .380 & .537 \\
\hline \multicolumn{7}{|c|}{ Amigos y apoyo social } \\
\hline Hombres & 90 & 55.56 & 72 & 44.44 & .120 & .728 \\
\hline Mujeres & 120 & 51.95 & 111 & 48.05 & 1.09 & .296 \\
\hline \multicolumn{7}{|l|}{ Entorno escolar } \\
\hline Hombres & 102 & 54.55 & 85 & 45.45 & .032 & .856 \\
\hline Mujeres & 117 & 48.35 & 125 & 51.65 & 1.34 & .247 \\
\hline \multicolumn{7}{|c|}{ Aceptación social (bullying) } \\
\hline Hombres & 57 & 45.60 & 68 & 54.40 & 9.50 & .002 \\
\hline Mujeres & 83 & 41.50 & 117 & 58.50 & 16.2 & .000 \\
\hline \multicolumn{7}{|c|}{ Recursos económicos } \\
\hline Hombres & 77 & 53.85 & 66 & 46.15 & .148 & .700 \\
\hline Mujeres & 85 & 51.83 & 79 & 48.17 & .379 & .538 \\
\hline
\end{tabular}

En cuanto a las relaciones intrafamiliares, se encontró una asociación estadísticamente significativa de la edad con la alta expresión en los hombres ( $\left.X^{2}(1) 11.22 ; p=.001\right)$ (Tabla $3)$.

En las Tablas 4 y 5 se muestra que la alta unión y apoyo familiar se asoció a mayor posibilidad de bienestar psicológico superior (OR 1.90, [1.19, 3.04]) y a 2 veces más posibilidad de mejor calidad de vida en relación con los padres y vida familiar (OR
2.33, [1.40, 3.87] en comparación con aquellos adolescentes que reportaron menor unión y apoyo familiar.

La alta posibilidad de expresarse en un entorno de respeto se asoció a casi 6 veces mayor posibilidad de calidad de vida superior en el dominio padres y vida familiar (OR 5.89, IC95\% [3.53, 9.83]), 2 veces mayor posibilidad de autonomía superior (OR 2.26, $[1.43,3.57]), 2$ veces mayor posibilidad de amigos y apoyo social superior (OR 2.33, 
[1.39, 3.89]), 2 veces mayor posibilidad de bienestar psicológico superior (OR 2.15, $[1.35,3.42])$ así como mayor posibilidad de entorno escolar superior (OR 1.85, [1.04, 3.29]), recursos económicos superiores (OR $1.64,[1.04,2.56])$ y autopercepción superior (OR 1.85, [1.15, 2.98]) en comparación con aquellos adolescentes que reportaron baja expresión.

Cuando los adolescentes reportaron una baja percepción de dificultades familiares, se encontró 3 veces mayor posibilidad de calidad de superior en el dominio relación con los padres y vida familiar bienestar (OR
3.08, IC95\% [1.95, 4.87]), 2 veces mayor posibilidad de autopercepción superior (OR 2.02, [1.33, 3.07]), así como mayor posibilidad de recursos económicos superiores (OR 1.68, [1.14, 2.50]), amigos y apoyo social superior (OR 1.61, [1.03, 2.52]), estado de ánimo superior (OR 1.91, [1.27, $2.86]$ ), bienestar psicológico superior ( $O R$ 1.51, [1.00, 2.27]), bienestar físico superior (OR 1.55, [1.04, 2.32]), entorno escolar superior (OR 1.80, [1.08, 3.00]) y aceptación social superior (OR 1.74, [1.16, 2.60]) en comparación con los adolescentes que se autolesionan y que reportaron mayor percepción de dificultades familiares.

TABLA 3.

Percepción de las relaciones intrafamiliares de los adolescentes mexicanos que se autolesionan sin intención suicida según sexo y sus diferencias por edad.

\begin{tabular}{|c|c|c|c|c|c|c|}
\hline \multirow{2}{*}{ Dimensiones ${ }^{a}$} & \multicolumn{2}{|c|}{$12-14$} & \multicolumn{2}{|c|}{$15-19$} & \multirow{2}{*}{$X^{2}$} & \multirow{2}{*}{$p$} \\
\hline & $\mathrm{n}$ & $\%$ & $\mathrm{n}$ & $\%$ & & \\
\hline \multicolumn{7}{|c|}{ Alta unión y apoyo } \\
\hline Hombres & 59 & 60.82 & 38 & 39.18 & 2.45 & .117 \\
\hline Mujeres & 61 & 53.04 & 54 & 46.96 & .601 & .438 \\
\hline \multicolumn{7}{|l|}{ Alta expresión } \\
\hline Hombres & 69 & 66.99 & 34 & 33.01 & 11.22 & .001 \\
\hline Mujeres & 66 & 54.10 & 56 & 45.90 & 1.23 & .267 \\
\hline \multicolumn{7}{|l|}{ Baja dificultad } \\
\hline Hombres & 42 & 52.50 & 38 & 47.50 & .268 & .604 \\
\hline Mujeres & 57 & 48.31 & 61 & 51.69 & .258 & .611 \\
\hline
\end{tabular}

TABLA 4.

Modelos de regresión logística para relaciones intrafamiliares y CVRS en adolescentes mexicanos que se autolesionan sin intención suicida, ajustados por variables de control.

\begin{tabular}{|c|c|c|c|c|c|c|c|c|c|c|}
\hline \multirow[t]{2}{*}{$\begin{array}{l}\text { Variables exposición y } \\
\text { controla }^{\text {Con }}\end{array}$} & \multicolumn{2}{|c|}{$\begin{array}{c}\text { Bienestar } \\
\text { físico } \\
\text { (Sup. }=1 \text { Inf. =0) }\end{array}$} & \multicolumn{2}{|c|}{$\begin{array}{c}\text { Bienestar } \\
\text { psicológico } \\
(\text { Sup.=1 Inf. }=0)\end{array}$} & \multicolumn{2}{|c|}{$\begin{array}{c}\text { Estado } \\
\text { de ánimo } \\
(\text { Sup. }=1 \text { Inf.=0) }\end{array}$} & \multicolumn{2}{|c|}{$\begin{array}{l}\text { Autopercepción } \\
\text { (Sup.=1 Inf.=0) }\end{array}$} & \multicolumn{2}{|c|}{$\begin{array}{c}\text { Autonomía } \\
\text { (Sup. }=1 \text { Inf. }=0)\end{array}$} \\
\hline & OR & $95 \%$ IC & OR & $95 \%$ IC & OR & $95 \%$ IC & OR & $95 \%$ IC & OR & $95 \%$ IC \\
\hline Alta unión y apoyo familiar & 1.40 & {$[0.88,2.23]$} & 1.90 & {$[1.19,3.04]$} & 1.44 & {$[0.90,2.29]$} & 0.90 & {$[0.56,1.45]$} & 1.53 & {$[0.97,2.42]$} \\
\hline Alta expresión & 1.50 & {$[0.95,2.39]$} & 2.15 & {$[1.35,3.42]$} & 1.49 & {$[0.94,2.36]$} & 1.85 & {$[1.15,2.98]$} & 2.26 & {$[1.43,3.57]$} \\
\hline Baja dificultad familiar & 1.55 & {$[1.04,2.32]$} & 1.51 & {$[1.00,2.27]$} & 1.91 & {$[1.27,2.86]$} & 2.02 & {$[1.33,3.07]$} & 1.31 & {$[0.88,1.95]$} \\
\hline Hombre & 2.85 & {$[1.95,4.16]$} & 2.77 & {$[1.89,4.08]$} & 2.39 & {$[1.63,3.50]$} & 2.25 & {$[1.53,3.30]$} & 1.87 & {$[1.29,2.72]$} \\
\hline Edad 15 a 19 años & 0.67 & {$[0.46,0.97]$} & 1.35 & {$[0.92,1.98]$} & 0.83 & {$[0.56,1.23]$} & 0.69 & {$[0.47,1.01]$} & 1.17 & {$[0.81,1.70]$} \\
\hline NSE medio alto y alto & 1.22 & {$[0.82,1.81]$} & 1.17 & {$[0.78,1.75]$} & 0.72 & {$[0.48,1.09]$} & 0.71 & {$[0.48,1.05]$} & 1.12 & {$[0.75,1.65]$} \\
\hline No discapacidad & 1.89 & {$[1.10,3.25]$} & 1.21 & {$[0.69,2.09]$} & 2.35 & {$[1.23,4.50]$} & 1.11 & {$[0.64,1.91]$} & 0.96 & {$[0.56,1.64]$} \\
\hline No abuso de alcohol & 1.28 & {$[0.77,2.13]$} & 1.31 & {$[0.78,2.20]$} & 1.52 & {$[0.85,2.71]$} & 1.39 & {$[0.84,2.31]$} & 0.99 & {$[0.60,1.64]$} \\
\hline No abuso de medicamentos & 0.88 & {$[0.42,1.87]$} & 0.97 & {$[0.46,2.05]$} & 1.54 & {$[0.65,3.61]$} & 1.07 & {$[0.51,2.27]$} & 2.05 & {$[0.98,4.28]$} \\
\hline
\end{tabular}


TABLA 5.

Modelos de regresión logística para relaciones intrafamiliares y CVRS en adolescentes mexicanos que se autolesionan sin intención suicida, ajustados por variables de control.

\begin{tabular}{|c|c|c|c|c|c|c|c|c|c|c|}
\hline \multirow[t]{2}{*}{$\begin{array}{c}\text { Variables exposición y } \\
\text { controla }^{\text {a }}\end{array}$} & \multicolumn{2}{|c|}{$\begin{array}{c}\text { Padres y } \\
\text { vida familiar } \\
(\text { Sup. }=1 \text { Inf. }=0)\end{array}$} & \multicolumn{2}{|c|}{$\begin{array}{c}\text { Amigos y } \\
\text { apoyo social } \\
(\text { Sup.=1 Inf. }=0)\end{array}$} & \multicolumn{2}{|c|}{$\begin{array}{c}\text { Entorno } \\
\text { escolar } \\
\text { (Sup. }=1 \text { Inf. =0) }\end{array}$} & \multicolumn{2}{|c|}{$\begin{array}{c}\text { Aceptación } \\
\text { social } \\
\text { (Sup. }=1 \text { Inf. =0) }\end{array}$} & \multicolumn{2}{|c|}{$\begin{array}{c}\text { Recursos } \\
\text { económicos } \\
(\text { Sup. }=1 \text { Inf. }=0)\end{array}$} \\
\hline & OR & $95 \%$ IC & OR & $95 \%$ IC & OR & $95 \%$ IC & OR & $95 \%$ IC & OR & $95 \%$ IC \\
\hline Alta unión y apoyo familiar & 2.33 & {$[1.40,3.87]$} & 1.15 & {$[0.69,1.91]$} & 1.63 & {$[0.91,2.92]$} & 1.21 & {$[0.76,1.90]$} & 1.48 & {$[0.94,2.33]$} \\
\hline Alta expresión & 5.89 & {$[3.53,9.83]$} & 2.33 & {$[1.39,3.89]$} & 1.85 & {$[1.04,3.29]$} & 1.52 & {$[0.96,2.40]$} & 1.64 & {$[1.04,2.56]$} \\
\hline Baja dificultad familiar & 3.08 & {$[1.95,4.87]$} & 1.61 & {$[1.03,2.52]$} & 1.80 & {$[1.08,3.00]$} & 1.74 & {$[1.16,2.60]$} & 1.68 & {$[1.14,2.50]$} \\
\hline Hombre & 2.18 & {$[1.42,3.34]$} & 0.86 & {$[0.58,1.28]$} & 1.41 & {$[0.90,2.20]$} & 0.71 & {$[0.49,1.03]$} & 1.52 & {$[1.06,2.20]$} \\
\hline Edad 15 a 19 años & 1.45 & {$[0.94,2.23]$} & 0.85 & {$[0.57,1.27]$} & 1.56 & {$[0.99,2.45]$} & 2.86 & {$[1.95,4.20]$} & 0.98 & {$[0.68,1.42]$} \\
\hline NSE medio alto y alto & 1.40 & {$[0.89,2.20]$} & 1.36 & {$[0.88,2.09]$} & 0.92 & {$[0.58,1.47]$} & 1.13 & {$[0.76,1.66]$} & 2.15 & {$[1.45,3.18]$} \\
\hline No discapacidad & 1.22 & {$[0.66,2.27]$} & 1.44 & {$[0.82,2.50]$} & 1.67 & {$[0.93,3.00]$} & 1.07 & {$[0.63,1.83]$} & 1.34 & {$[0.79,2.28]$} \\
\hline No abuso de alcohol & 1.87 & {$[1.04,3.35]$} & 0.75 & {$[0.43,1.30]$} & 2.14 & {$[1.24,3.71]$} & 1.38 & {$[0.83,2.31]$} & 0.71 & {$[0.43,1.19]$} \\
\hline No abuso de medicamentos & 1.75 & {$[0.75,4.08]$} & 3.34 & {$[1.60,6.99]$} & 1.19 & {$[0.54,2.63]$} & 1.71 & {$[0.82,3.57]$} & 1.37 & {$[0.66,2.82]$} \\
\hline
\end{tabular}

\section{DISCUSIÓN}

El propósito de este estudio fue analizar la asociación de la calidad de vida con la percepción de las relaciones intrafamiliares en adolescentes que se autolesionan sin intención suicida. Se encontró que la percepción positiva de las relaciones intrafamiliares se asocia a calidad de vida superior en la mayoría de las dimensiones. La baja dificultad familiar fue la dimensión que más se asoció a calidad de vida superior en nueve de los diez dominios, mientras que la alta capacidad de expresarse se asoció a siete dominios y alta unión y apoyo a dos dominios de CVRS.

Cuando se comparó la CVRS de acuerdo a los grupos de edad, se encontró que en los adolescentes de mayor edad, la CVRS fue menor en los dominios bienestar físico para los hombres y en la autopercepción para las mujeres, lo que concuerda con estudios previos (González et al., 2016) sin embargo, llama la atención que los adolescentes de 12 a 14 años reportaron menor aceptación social que los de 15 a 19 años, tanto para hombres como para mujeres. Lo anterior podría indicar que el bullying es más frecuente a menor nivel educativo, tendencia que fue reportada al encontrar mayor acoso escolar en primarias (primera etapa de educación básica que dura 6 años) que en secundarias (etapa posterior a la primaria que dura 3 años) de México (Aguilera, Muñoz \& Orozco, 2007). Otra posible explicación es que, a mayor nivel educativo se reste importancia a las conductas de acoso escolar y que sean percibidas como una conducta normalizada. Sobre las relaciones intrafamiliares, se encontró que durante la adolescencia tardía (15 a 19 años) una menor cantidad de hombres reportaron alta expresión dentro de la familia, lo que consideramos puede deberse a que, durante esta etapa de desarrollo los adolescentes ya cuentan con mayor seguridad y claridad de sus propias opiniones (UNICEF, 2011) aunado a que los hombres suelen actuar de manera más agresiva y abierta en su expresión de inconformidades (Hurlock, 1987) lo que pudiera generar conflictos con otros integrantes de la familia, disminuyendo la percepción del adolescente de su capacidad de expresarse en este contexto sin ser reprendido.

La alta unión y apoyo familiar se asoció al bienestar psicológico superior de los adolescentes, lo que pudiera deberse a que el entorno familiar cumple una función primordial en el aprendizaje del 
procesamiento emocional, es decir, la manera en que los niños y los adolescentes experimentan, controlan y expresan sus emociones, por lo que hogares donde el clima emocional es frío, negligente o no existe apoyo entre los miembros, pueden convertirse en un entorno de riesgo (Repetti, Taylor \& Seeman, 2002). Asimismo, la baja unión y convivencia entre los miembros de una familia, pudieran ser percibidos por el adolescente como una situación indeseable, generando sentimientos de tristeza y abandono; este resultado coincide con estudios previos donde concluyeron que los conflictos familiares incrementan la probabilidad de un estado de ánimo deprimido en los adolescentes (Sigfusdottir, Asgeirsdottir, Sigurdsson \& Gudjonsson, 2011) y que existe mayor regulación emocional adaptativa en un ambiente positivo y unido, en el que los padres son expresivos, cálidos y sensibles a los afectos del niño (Fosco \& Grych, 2013). Además, existe evidencia de que el clima emocional de la familia afecta la regulación emocional del adolescente a partir del estilo parental, del apego en la relación, la expresividad y la relación entre los miembros de una familia (Morris, Silk, Steinberg, Myers, \& Robinson 2007), lo que nos permite afirmar que la percepción de unión y apoyo familiar es un factor primordial en el bienestar psicológico del adolescente, especialmente si consideramos que los adolescentes con autolesión no suicida, reportan mayor malestar psicológico que la población en general.

Por otro lado, la dimensión expresión definida en este estudio como la posibilidad del adolescente de comunicar sus emociones, ideas y sucesos en un ambiente de respeto (Rivera \& Andrade, 2010) se asoció a la calidad de vida superior, lo que podría indicar que para el adolescente es sumamente significativo darse cuenta que puede opinar y exteriorizar lo que siente sin sentirse juzgado o rechazado, saberse escuchado y respetado entre sus padres y hermanos. Cuando los adolescentes reportaron alta expresión, obtuvieron bienestar psicológico superior; al respecto Barber (1996) sustentó que cuando los padres restringen, invalidan o manipulan la experiencia y expresión emocional de sus hijos, aparecen síntomas de internalización, como la agresión e hiperactividad y de externalización, tal como el aislamiento social y emociones negativas como la ansiedad. Más aún, el abuso psicológico por parte de los padres puede manifestarse como conductas repetidas de abuso verbal, castigos severos no físicos o amenazas de abuso de un padre a su hijo, lo que conlleva a sentirse inútil, defectuoso, no amado, indeseado, en peligro, o que su valor depende de satisfacer las necesidades de otra persona, lo que está relacionado con problemas de salud mental (Rizvi \& Najam, 2014). También se sabe que la relación entre padres e hijos afecta el autoconcepto del adolescente, lo que a su vez afecta su integración al grupo de pares y sus relaciones interpersonales, es decir, el concepto de sí mismo juega un papel mediador entre el estilo de crianza de los padres y las relaciones satisfactorias con los pares, ya que un padre afectuoso, que acepta y afirma emocionalmente a su hijo, le transmite un sentimiento de valía, se ve a sí mismo como digno de cuidado, amor y atención que es la base de una autoestima sana y ofrece un modelo de relación donde los otros son vistos como confiables y disponibles; además otorga seguridad al niño de explorar los límites personales y aptitudes, que es importante para desarrollar un sano autoconcepto (Deković \& Meeus, 1997). En este sentido, nuestros resultados coinciden con estas afirmaciones, ya que los adolescentes con alta expresión, reportaron también autopercepción superior y calidad de vida superior en entorno escolar, y amigos y apoyo social.

La alta expresión también se asoció con autonomía superior, lo que pudiera indicar que en las familias donde existe mayor oportunidad para el adolescente de revelarse emocional o ideológicamente, existe también 
más fomento a la independencia y a asumirse como individuo, así como mayor libertad para tomar sus propias decisiones. Si bien uno de los principales conflictos entre padres e hijos adolescentes es el tema de la autoridad parental y la autonomía del menor, los resultados de estudios previos indican que éstos pueden tener una base cultural y que la autonomía, cuando es percibida como una tarea de desarrollo del adolescente, aunque genera distanciamiento en la relación con los padres y mayor conflicto con las madres, tiende a disminuir y a superarse a medida que el adolescente crece y que es capaz de expresar abiertamente su desacuerdo con los padres en aspectos personales (Fuligni, 1998). La importancia de adquirir autonomía durante la adolescencia, ha sido analizada por Cooper, Grotevant y Condon (1983) desde la perspectiva familiar y han encontrado que la individualidad y la conexión en la comunicación familiar, facilita la formación de la identidad, que se expresa en elegir una ocupación, roles sexuales, creencias políticas, religiosas o filosóficas, así como relaciones interpersonales y en la habilidad de tomar roles, lo que es posible cuando la familia dota de libertad $y$ capacidad al adolescente de explorar diversas posibilidades en su vida, a la vez que percibe una base segura de conexión y apoyo con los padres, en la que incluso expresiones respetuosas de desacuerdo entre los padres, son vistas como diferencias de opinión que pueden ser expresadas, lo que provee un equilibrio entre individualidad y conexión familiar.

La variable menos dificultades, analizada en este estudio como la percepción de aspectos considerados por el adolescente como problemáticos, indeseables 0 conflictivos en la familia, se asoció a alta calidad de vida en nueve dominios; en un estudio previo, los adolescentes refirieron que experiencias de un ambiente emocionalmente cálido, una comunicación abierta, involucramiento familiar, posibilidades de relaciones externas y un sentido de valía personal en la familia eran situaciones que les generaba bienestar (Joronen \& Astedt-Kurki, 2005). Respecto a su asociación con el bienestar físico superior, Katrín (2013) reportó que la actividad física disminuye el impacto negativo de vivir situaciones de conflicto familiar. Otro estudio en adolescentes con enfermedad inflamatoria intestinal (Reed-Knight, Lewis \& Blount, 2011) encontró una mejor adhesión al tratamiento cuando existía mayor participación materna y menor conflicto percibido, lo que consideramos es un indicio de que los conflictos familiares pudieran afectar la sensación del adolescente de sentirse sano y con energía, además del seguimiento a diversos tratamientos en los adolescentes con padecimientos, entre los que se pudiera incluir a la autolesión no suicida. Además, nuestro estudio encontró asociación entre menores dificultades familiares y bienestar psicológico y estado de ánimo superior, lo que coincide con los resultados de un estudio que analizó durante un año la relación entre apoyo familiar, conflicto familiar y sintomatología depresiva en adolescentes y en sus madres y reveló que los ambientes familiares con mayor conflicto y menos apoyo se asociaron a mayor sintomatología depresiva (Sheeber, Hops, Alpert, Davis, \& Andrews 1997). Por su parte Fosco y Grych (2013), encontraron que los niños entre 8 y12 años expuestos a conflictos parentales más hostiles $y$ frecuentes y a ambientes familiares críticos, tendían a presentar una regulación emocional menos adaptativa. Incluso existe evidencia de que la separación o divorcio de los padres es mejor para la salud emocional de los niños, pues vivir en situación de constante conflicto familiar causa estrés emocional (Jekielek, 1998) Otro estudio concluyó que un grado elevado y continuo de conflicto en la familia se asoció a cambios en el estado de ánimo, ansiedad y síntomas físicos de malestar en los adolescentes, además de un menor bienestar que quienes vivían situación de divorcio de los padres 0 bajo conflicto familiar (Mechanic \& Hansell, 1989). 
En relación a la autopercepción superior, consideramos que el adolescente al encontrarse en un proceso de construcción de su identidad, es vulnerable a valorarse a sí mismo en función del juicio que realice de su familia, de tal manera que, si evalúa su ambiente familiar como agradable, tienda a percibirse de manera positiva y a sentirse seguro y satisfecho consigo mismo. Un estudio que examinó la relación entre el ambiente familiar, la personalidad de los padres y la salud mental de los adolescentes encontró que las familias perturbadas contribuyeron a la ansiedad de los adolescentes, a la incapacidad para compartir problemas personales, la interferencia de los padres en los asuntos personales y a la presión académica; además de que los rasgos de los padres influyeron negativamente en la salud mental de los adolescentes por ejemplo, ansiedad, bajo ajuste, autoconcepto y confianza en sí mismos (Deb, McGirr, Bhattacharya, \& Sun, 2015). Otro estudio concluyó que la exposición infantil al abuso de alcohol y a la violencia familiar afectaban el funcionamiento psicosocial en la adolescencia, con mayor consumo de sustancias, trastornos de la conducta y baja autoestima (Ritter, Stewart, Bernet, Coe, \& Brown, 2002).

Otros dominios asociados a una baja dificultad familiar fue el de amigos y apoyo social y entorno escolar superior. Estos resultados corroboran lo encontrado por Repetti, Taylor y Seeman (2002) en el sentido de que las habilidades sociales y para construir relaciones de apoyo fuera de la familia, son afectadas por el hecho de crecer en un entorno familiar frío, negligente o carente de afecto, pues a partir de sus relaciones familiares, el adolescente aprende a comportarse y relacionarse con los demás. En cuanto a la asociación de recursos económicos superiores y baja dificultad familiar y alta expresión, coincidimos con Conger et al. (1994) quienes confirmaron un modelo en el cual la presión económica de los padres incrementa la irritabilidad y los conflictos sobre el dinero, tanto con la pareja como con los hijos, lo que incrementa el riesgo de problemas emocionales y de conducta en los adolescentes.

En cuanto a la asociación de baja dificultad familiar con aceptación social superior, consideramos que vivir en un ambiente familiar cálido, donde le es permitido manifestar sus ideas, sus emociones y compartir sus vivencias cotidianas en un ambiente de respeto, pudiera promover su sentido de protección parental y de autoprotección frente a la intimidación y acoso, además de prevenir la repetición de estos eventos, al comunicar a sus figuras de autoridad el acoso del que es víctima, consciente de que será escuchado y respetado. Bowes et al. (2009) reportó en niños una asociación entre realizar pocas actividades con la madre y ser víctima de intimidación, ya que los padres de las víctimas suelen estar menos involucrados con sus hijos. También experimentar maltrato en el hogar puede generar riesgo de ser víctima de acoso, debido a los signos de negligencia o marcas visibles de daño físico, mientras que otro estudio concluyó que la calidez materna y con los hermanos, así como un clima emocional positivo en el hogar fue un factor que promovió la resiliencia ante la intimidación, por lo que estos factores familiares se consideran de protección ante el acoso escolar (Bowes, Maughan, Caspi, Moffitt, \& Arseneault, 2010).

Este estudio tiene limitaciones que deben ser consideradas. Una de éstas es que el diseño transversal permite dar cuenta únicamente de la magnitud de la asociación entre las variables, por lo que no es posible asumir relaciones de causalidad. Otro aspecto importante, es que se consideró el reporte de al menos una conducta de autolesión no suicida para ser incluido en los análisis, sin embargo, no se evaluó la severidad o la frecuencia de autolesión. Finalmente, este estudio aporta evidencia de que las relaciones intrafamiliares están asociadas a la CVRS de los adolescentes 
mexicanos que se autolesionan, incluso cuando se consideran variables como el sexo, la edad, el nivel socioeconómico, la discapacidad y el abuso de sustancias. A partir de estos hallazgos, se sugiere incorporar el análisis de las relaciones intrafamiliares en la evaluación inicial, tratamiento y seguimiento de los adolescentes que se autolesionan sin intención suicida.

\section{REFERENCIAS}

Aguilera, A., Muñoz, G., \& Orozco A. (2007). Disciplina, violencia y consumo de sustancias nocivas a la salud en primarias y secundarias, México: Instituto Nacional para la Evaluación de la Educación.

Albores-Gallo, L., Méndez-Santos, J. L., García Luna, A. X., DelgadilloGonzález, Y., Chávez-Flores, C. I., Martínez, O. L. (2014). Autolesiones sin intención suicida en una muestra de niños y adolescentes de la ciudad de México. Actas Españolas de Psiquiatría, 42(4), 159-68.

Baetens, I., Claes, L., Martin, G., Onghena, P., Grietens, H., Van Leeuwen, K., \& Griffith, J. W. (2014). Is Nonsuicidal Self-Injury Associated With Parenting and Family Factors? The Journal of Early Adolescence, 34(3), 387-405. https://doi.org/10.1177/0272431613494 006

Baña, C.M. (2015). El rol de la familia en la calidad de vida y la autodeterminación de las personas con trastorno del espectro del autismo. Ciencias Psicológicas, $\quad$ 9(2), 323-336. Recuperado de http://www.scielo.edu.uy/pdf/cp/v9n2/v9 n2a09.pdf

Barber, B. K. (1996). Parental psychological control: Revisiting a neglected construct. Child Development, 67, 3296-3319.

Benjet, C., González-Herrera, I., CastroSilva, E., Méndez, E., Borges, G.,
Casanova, L., \& Medina-Mora, M. E. (2017). Non-suicidal self-injury in Mexican young adults: Prevalence, associations with suicidal behavior and psychiatric disorders, and DSM-5 proposed diagnostic criteria. Journal of Affective Disorders, 215, 1-8. doi:10.1016/j.jad.2017.03.025

Bowes, L., Arseneault, L., Maughan, B., Taylor, A., Caspi, A., \& Moffitt, T. E. (2009). School, Neighborhood, and Family Factors Are Associated With Children's Bullying Involvement: A Nationally Representative Longitudinal Study. Journal of the American Academy of Child \& Adolescent Psychiatry, 48(5), 545-553. https://doi.org/10.1097/CHI.0b013e318 $19 \mathrm{cb} 017$

Bowes, L., Maughan, B., Caspi, A., Moffitt, T. E., \& Arseneault, L. (2010). Families promote emotional and behavioural resilience to bullying: evidence of an environmental effect. Journal of Child Psychology and Psychiatry, 51(7), 809817. https://doi.org/10.1111/j.14697610.2010.02216.x

Brunner, R., Kaess, M., Parzer, P., Fischer, G., Carli, V., Hoven, C. W.,... Wasserman, D. (2014). Life- time prevalence and psychosocial correlates of adolescent direct self-injurious behavior: A comparative study of findings in 11 European countries. Journal of Child Psychology and Psychiatry, 55(4), 337-348. doi:10.1111/jcpp.12166

Carvalho, C. B., Nunes, C., Castilho, P., da Motta, C., Caldeira, S., \& Pinto-Gouveia, J. (2015). Mapping non suicidal selfinjury in adolescence: Development and confirmatory factor analysis of the impulse, self-harm and suicide ideation questionnaire for adolescents (ISSIQA). Psychiatry Research, 227(2-3), 238-245.

https://doi.org/10.1016/j.psychres.2015. 01.031

Chávez-Flores, Y.V. Hidalgo-Rasmussen, C.A., \& González-Betanzos, F. (2017). 
Adaptación transcultural del cuestionario ISSIQ-A para evaluar impulsividad, autolesión no suicida e ideación suicida en adolescentes mexicanos. Terapia psicológica. Enviado 22 de diciembre de 2017.

Conger, R. D., Ge, X., Elder, G. H., Lorenz, F. O., \& Simons, R. L. (1994). Economic Stress, Coercive Family Process, and Developmental Problems of Adolescents. Child Development, 65(2), 541-561.

https://doi.org/10.1111/j.1467-

8624.1994.tb00768.x

Cooper, C. R., Grotevant, H. D., \& Condon, S. M. (1983). Individuality and connectedness in the family as a context for adolescent identity formation and role-taking skill. New Directions for Child and Adolescent Development, 1983(22), 43-59. https://doi.org/10.1002/cd.2321983220 5

Deb, S., McGirr, K., Bhattacharya, B., \& Sun, J. (2015). Role of Home Environment, Parental Care, Parents Personality and Their Relationship to Adolescent Mental Health. Journal of Psychology \& Psychotherapy, 5(6), 1-8. https://doi.org/10.4172/21610487.1000223

Deković, M., \& Meeus, W. (1997). Peer relations in adolescence: effects of parenting and adolescents' selfconcept. Journal of Adolescence, 20(2), 163-176.

https://doi.org/10.1006/jado.1996.0074

Di Pierro, R., Sarno, I., Perego, S., Gallucci, M., \& Madeddu, F. (2012). Adolescent nonsuicidal self-injury: The effects of personality traits, family relationships and maltreatment on the presence and severity of behaviours. European Child and Adolescent Psychiatry, 21(9), 511520. https://doi.org/10.1007/s00787012-0289-2

Fosco, G. M., \& Grych, J. H. (2013). Capturing the family context of emotion regulation: A family systems model comparison approach. Journal of Family
Issues, $\quad 34(4), \quad$ 557-578. https://doi.org/10.1177/0192513X12445 889

Fondo de las Naciones Unidas para la Infancia. UNICEF. (2011). Estado mundial de la infancia 2011. La adolescencia: una época de oportunidades. Recuperado de https://www.unicef.org/spanish/sowc20 11/pdfs/SOWC-2011-Main Report_SP_02092011.pdf

Fuligni, A. J. (1998). Authority, autonomy, and parent-adolescent conflict and cohesion: a study of adolescents from Mexican, Chinese, Filipino, and European backgrounds. Developmental Psychology, 34(4), 782-92. Recuperado de http://www.ncbi.nlm.nih.gov/pubmed/96 81270

González, A. E., Martínez N. V., Molina G. T., Myriam, G. L., Sepúlveda P. R., Molina C. R., \& Hidalgo-Rasmussen, C. (2016). Diferencias de género en la calidad de vida relacionada con la salud en adolescentes escolarizados chilenos. Revista médica de Chile, 144 (3). Recuperado de http://dx.doi.org/10.4067/S003498872016000300004

Grey, M., Boland, E. A., Yu, C., SullivanBolyai, S. \& Tamborlane, W.V. (1998). Personal and family factors associated with quality of life in adolescents with diabetes. Diabetes Care, 21(6), 909914. Recuperado de https://doi.org/10.2337/diacare.21.6.90 9

Guedes, D.P., Astudillo, H.A.V., Morales, J.M.M., del Campo Vecino, J., Pires Júnior, R. (2014). Calidad de vida relacionada con la salud de adolescentes latinoamericanos. Revista Panamericana de Salud Pública, 35(1), 46-52.

Herzer, M., Denson, L.A., Baldassano, R.N., \& Hommel, K.A. (2011). Patient and parent psychosocial factors associated with health-related quality of life in pediatric inflammatory bowel disease. 
Journal of pediatric gastroenterology and nutrition, 52(3), 295-9. Recuperado de

https://www.ncbi.nlm.nih.gov/pmc/articl es/PMC3058639/

Hidalgo-Rasmussen, C. A., Rajmil, L., \& Montaño, R. (2014). Adaptación transcultural del cuestionario KIDSCREEN para medir calidad de vida relacionada con la salud en población argentina de 8 a 18 años. Ciéncia \& Saude Coletiva, 19(7), 2215-2224. https://doi.org/10.1590/141381232014197.09682013

Hurlock, E. B. (1987). Psicología de la adolescencia. México, D.F.: Paidós.

Jekielek, S. M. (1998). Parental Conflict, Marital Disruption and Children's Emotional Well-Being. Social Forces, 76(3), 905-936. https://doi.org/10.1093/sf/76.3.905

Jiménez-Iglesias, A., Moreno, C., Ramos, P., \& Rivera, F. (2015). What family dimensions are important for healthrelated quality of life in adolescence? Journal of Youth Studies, 18(1), 53-67. https://doi.org/10.1080/13676261.2014. 933191

Jordán, J. M., Molina, S. J., \& Pillon, S. C. (2009). Uso de drogas y factores de riesgo entre estudiantes de enseñanza media. Revista Latino-Americana de Enfermagem, 17(2), 109-115. http://www.scielo.br/pdf/rlae/v17n2/es_ 17.pdf

Joronen, K., \& Åstedt-Kurki, P. (2005). Familial contribution to adolescent subjective well-being. International Journal of Nursing Practice, 11(3), 125133. https://doi.org/10.1111/j.1440172X.2005.00509.X

Mayer Villa, P., Morales Gordillo, N., Victoria Figueroa, G., Ulloa Flores, R. E. (2016). Adolescentes con autolesiones e ideación suicida: un grupo con mayor comorbilidad y adversidad psicosocial. Salud Pública de México, 58, 335-336.

Meade, T. \& Dowswell, E. (2016). Adolescents' health-related quality of life (HRQoL) changes over time: a three year longitudinal study. Health and Quality of Life Outcomes 14(14). https://doi.org/10.1186/s12955-0160415-9

Mechanic, D., \& Hansell, S. (1989). Divorce, family conflict, and adolescents' wellbeing. Journal of Health and Social Behavior, 30(1), 105-16. Recuperado de

http://www.ncbi.nlm.nih.gov/pubmed/27 23376

Morris, A. S., Silk, J. S., Steinberg, L., Myers, S. S., \& Robinson, L. R. (2007). The Role of the Family Context in the Development of Emotion Regulation. Social Development, 16(2), 361-388. https://doi.org/10.1111/j.14679507.2007.00389.x

Muehlenkamp, J. J., Claes, L., Havertape, L., \& Plener, P. L. (2012). International prevalence of adolescent non- suicidal self-injury and deliberate self-harm. Child and Adolescent Psychiatry and Mental Health, 6(1), 10. https://doi.org/10.1186/1753-2000-6-10

Nock, M. K. (2010). Self-Injury. Annual Review of Clinical Psychology, 6(1), 339-363.

https://doi.org/10.1146/annurev.clinpsy. 121208.131258

Organización Mundial de la Salud. (2017). Adolescentes: riesgos para la salud y soluciones. Recuperado de http://www.who.int/mediacentre/factshe ets/fs345/es/

Organización Mundial de la Salud. (2017). Cada año fallecen más de 1,2 millones de adolescentes por causas que, en su mayor parte, podrían evitarse. Recuperado de http://www.who.int/mediacentre/news/r eleases/2017/yearly-adolescentdeaths/es/

Organización Panamericanada de la Salud. (2008). Jóvenes: opciones y cambios. Promoción de conductas saludables en la adolescencia. Washington, D.C: Organización Panamericana de la Salud.

Ravens-Sieberer, U., Herdman, M., Devine, 
J., Otto, C., Bullinger, M., Rose, M., \& Klasen, F. (2014). The European KIDSCREEN approach to measure quality of life and well-being in children: Development, current application, and future advances. Quality of Life Research, 23(3), 791-803. https://doi.org/10.1007/s11136-0130428-3

Reed-Knight, B., Lewis, J. D., \& Blount, R. L. (2011). Association of Disease, Adolescent, and Family Factors with Medication Adherence in Pediatric Inflammatory Bowel Disease. Journal of Pediatric Psychology, 36(3), 308-317. https://doi.org/10.1093/jpepsy/jsq076

Repetti, R. L., Taylor, S. E., \& Seeman, T. E. (2002). Risky families: Family social environments and the mental and physical health of offspring. Psychological Bulletin, 128(2), 330-366. https://doi.org/10.1037//00332909.128.2.230

Revicki, D. A., Cella, D., Hays, R. D., Sloan, J. A., Lenderking, W. R., \& Aaronson, N. K. (2006). Responsiveness and minimal important differences for patient reported outcomes. Health Qual Life Outcomes, $\quad 4(1), \quad 70$ http://www.ncbi.nlm.nih.gov/pubmed/17 005038

Ritter, J., Stewart, M., Bernet, C., Coe, M., \& Brown, S. A. (2002). Effects of childhood exposure to familial alcoholism and family violence on adolescent substance use, conduct problems, and selfesteem. Journal of Traumatic Stress, 15(2), 113-122. https://doi.org/10.1023/A:10148039072 34

Rivas-Torres, R \& Bianchi-Águila, R. (1991). El nivel socioeconómico en la investigación. Revista de la Asociación Mexicana de Enfermedades metabólicas y de Obesidad, 2(1), 44-5.

Rivera, M. E., \& Andrade, P. (2010). Escala de evaluación de las Relaciones Intrafamiliares. Uaricha Revista de Psicología, 14, 12-29.

Rizvi, S. F. I., \& Najam, N. (2014). Parental psychological abuse toward children and mental health problems in adolescence. Pakistan Journal of Medical Sciences, 30(2), 256-260. https://doi.org/10.12669/pjms.302.4593

Sheeber, L., Hops, H., Alpert, A., Davis, B., \& Andrews, J. (1997). Family support and conflict: Prospective relations to adolescent depression. Journal of Abnormal Child Psychology, 25(4), 333344.

https://doi.org/10.1023/A:10257685044 15

Sigfusdottir, I. D., Asgeirsdottir, B. B., Sigurdsson, J. F., \& Gudjonsson, G. H. (2011). Physical activity buffers the effects of family conflict on depressed mood: A study on adolescent girls and boys. Journal of Adolescence, 34(5), 895-902.

https://doi.org/10.1016/j.adolescence.2 011.01 .003

Valenzuela, M. T., Ibarra R., A. M., Zubarew G., T., \& Correa, M. L. (2013). Prevención de conductas de riesgo en el Adolescente: rol de familia. Index de Enfermería, 22(1-2), 50-54. https://doi.org/10.4321/S113212962013000100011

Whitlock, J., Muehlenkamp, J., Eckenrode, J., Purington, A., Abrams, G. B., Barreira, P., \& Kress, V. (2012). Nonsuicidal Self-injury as a Gateway to Suicide in Young Adults. Journal of Adolescent

Health. https://doi.org/10.1016/j.jadohealth.201 2.09 .010

Zetterqvist, M. (2015). The DSM-5 diagnosis of nonsuicidal self-injury disorder: a review of the empirical literature. Child and Adolescent Psychiatry and Mental Health, $\quad 9(1), \quad 31$. https://doi.org/10.1186/s13034-0150062-7 